C'est malheureusement dans l'air du temps de se prévaloir des possibilités de l'informatique pour amasser des quantités de données à la destination incertaine, ou pour tenter de contrôler tout ce que les esprits les plus obsessionnels peuvent imaginer contrôler.
Un nouvel exemple en est fourni ci-dessous - merci aux auteur-e-s de cette présentation éloquente.

Dr Jacques de Haller, Président de la FMH

\title{
Lorsque le diagnostic devient un bien commun...
}

Dernièrement, lors du eHealth-Summit, Hanspeter Thür, Préposé fédéral à la protection des données, a rapporté qu'aux Etats-Unis, les premiers changements de nom avaient été accordés au motif que ce serait la seule manière pour la personne concernée de se débarrasser des effets collatéraux de son profil sur Facebook. Lors de ce même congrès, des sources normalement bien informées ont annoncé la récente installation en Suisse d'entreprises dont l'activité consiste à se procurer des données de santé pour les revendre à des acquéreurs intéressés: banques, assurances-vie, employeurs. Un modèle d'entreprise qui ne doit pas nous étonner, il a en effet déjà une certaine tradition aux Etats-Unis.

Et que fait la Suisse pour protéger efficacement les données de santé contre des abus? Lorsqu'il s'est penché il y a trois ans sur le financement hospitalier dans le cadre de la révision de la LAMal, le Parlement a certes, après un débat approfondi, refusé la transmission automatique des diagnostics [1]. Toutefois, dans le cadre des mesures urgentes pour endiguer l'évolution des coûts, la formulation suivante est à nouveau proposée au vote final:

LAMal art. 42, al. 3bis «Les fournisseurs de prestations doivent faire figurer dans la facture selon l'al. 3 les diagnostics sous forme codée, conformément à la classification internationale des maladies contenue dans l'édition suisse correspondante publiée par le département compétent. Le Conseil fédéral édicte des dispositions détaillées sur la collecte, le traitement et la transmission des données, dans le respect du principe de la proportionnalité.»

Nous sommes donc sur le point

- premièrement, avec le nouveau numéro d'assurance sociale, d'identifier [2] clairement le citoyen tout au long de sa vie sur les factures de médecin et d'hôpital et

- deuxièmement, de fournir en même temps le diagnostic à tous ceux qui liront la facture.

Dans les deux cas, le législateur s'est laissé entraîner à des réactions rapides et irréfléchies. L'utilisation du nouveau numéro d'assurance sociale pour la facturation dans le cadre de la LAMal a été justifiée par des arguments relatifs à l'efficacité, alors que santésuisse a déclaré après coup que les assureurs n'ont besoin ni du $\mathrm{n}^{\circ}$ de carte d'assuré ni du $\mathrm{n}^{\circ} \mathrm{AVS}$ pour identifier les assurés [3]. L'indication du diagnostic sur la facture a été vendue au Parlement avec l'argument d'un meilleur contrôle des coûts - mais là aussi sans preuve à l'appui.

En revanche, les dommages potentiels sont évidents. Les péripéties autour des $\mathrm{CD}$ des données fiscales l'ont montré.
Il est toujours plus rarement possible de localiser l'origine d'une transmission non autorisée de données. Il en va de même pour le domaine de la santé. A l'ère de la documentation électronique et de l'échange électronique des données, le nombre de personnes ayant accès à des informations confidentielles est en constante augmentation et il devient toujours plus difficile de protéger efficacement les données. La présence du diagnostic sur la facture agrandit à nouveau clairement le cercle des personnes qui ont accès aux données sensibles des patients et accroît donc aussi le risque de leur divulgation non autorisée.

A cela s'ajoute le fait que les membres de la famille, mais le cas échéant également l'aide sociale, et les autorités tutélaires ou fiscales, ne devraient pas toutes apprendre qui a eu recours à une interruption volontaire de grossesse ou pourquoi un tel est allé consulter un psychiatre. Pourtant, c'est exactement ce qui va se passer si le diagnostic doit être indiqué sur la facture.

Pour pouvoir examiner l'efficacité des hôpitaux et des médecins en matière de coûts, il faut des données agrégées au niveau de l'hôpital ou du médecin. Pour la compensation des risques, il faut des données agrégées au niveau des assureurs ou du modèle d'assurance. Par contre, dans aucun de ces cas l'indication non anonymisée des données individuelles sur la facture n'est appropriée, nécessaire ou même proportionnée. De plus, si la disposition légale susmentionnée devait entrer en vigueur et être appliquée, le Parlement et le Conseil fédéral porteraient un sérieux coup à la promotion de la cybersanté en Suisse. En effet, la réussite de la mise en œuvre des applications eHealth n'est pas envisageable sans la confiance des patients en une protection efficace des données.

Dr sc. hum. Judith Wagner, responsable du domaine eHealth FMH

Hanspeter Kuhn, avocat, secrétaire général adjoint FMH

1 AB 2007 N 442-446. Voir à ce sujet: Lang G, Kuhn H, Meyer B, Ingenpass P, Wagner J. La révision du codage externe et professionnelle permet de garantir la protection des données. Bull Méd Suisses. 2010;91(34):1265-8.

2 Cf. Art. 42a, al. 1 et 2 LAMal. Le nouveau numéro d'assurance sociale doit être valable pour toute la vie et la personne doit être clairement identifiable, voir le message relatif à la modification de la loi fédérale sur l'assurance vieillesse et survivants (Nouveau numéro d'assuré AVS) du 23 novembre 2005, FF 2006 515ss.

3 Circulaire 07/2010 de santésuisse aux assureurs, 2 février 2010. 